\title{
Glandular Papillomatosis
}

National Cancer Institute

\section{Source}

National Cancer Institute. Glandular Papillomatosis. NCI Thesaurus. Code C65198.

Multifocal neoplastic proliferations of the glandular epithelium displaying a papillary

pattern. 